Original Paper http://ajol.info/index.php/ijbcs http://indexmedicus.afro.who.int

\title{
Déterminants de l'accès aux crédits agricole par les producteurs d'anacarde au nord-Bénin
}

\author{
Jean-Marie S. AWO ${ }^{1,2^{*}}$, Nouroudine OLLABODÉ ${ }^{1}$ et Jacob A. YABI ${ }^{1,2}$ \\ ${ }^{1}$ Laboratoire d'Analyse et de Recherches sur les Dynamiques Economiques et Sociales (LARDES), \\ Département d'Economie et de Sociologie Rurales, Faculté d'Agronomie, Université de Parakou BP 123 - \\ Parakou, République du Bénin. \\ ${ }^{2}$ Ecole Doctorales des Sciences Agronomiques et de l'Eau, Université de Parakou, Bénin. \\ "Auteur correspondant ; E-mail: awofils@gmail.com ; Tél : (+229) 95537036
}

\begin{tabular}{lll}
\hline Received: 05-06-2021 & Accepted: 23-08-2021 & Published: 31-08-2021 \\
\hline RÉSUMÉ & &
\end{tabular}

Pour sécuriser les revenus agricoles des producteurs béninois, des mécanismes de financement sont mis en place dans la production d'anacarde. La présente étude identifie les déterminants de l'accès au financement par les producteurs d'anacarde. A cet effet, des données socioéconomiques ont été collectées par enquête auprès de 160 producteurs de noix de cajou choisis aléatoirement dans deux communes du Nord-Bénin. La statistique descriptive a été utilisée pour caractériser les types de financement. Un modèle de régression Logit binaire a été estimé pour déterminer les variables influençant l'accès aux crédits par les producteurs d'anacarde. Les principaux résultats de cette étude indiquent trois types de financement sont obtenus par les producteurs d'anacarde et financés par divers acteurs. Le sexe, l'âge, l'appartenance à une organisation, les contacts avec les services de vulgarisation et la formation à la production d'anacarde déterminent l'accès au crédit agricole au nord du Bénin. Enfin, la promotion du financement agricole dans la zone d'étude permettra de réguler la capacité à introduire l'agroforesterie dans l'exploitation agricole dans le contexte d'amélioration des conditions de vie et la protection des sols.

(C) 2021 International Formulae Group. All rights reserved.

Mots clés: Financement agricole, mécanisme de financement, N’Dali, Tchaourou.

\section{Determinants of access to agricultural credits by casnacard growers in north Benin}

\begin{abstract}
In order to secure the agricultural income of producers in Benin, agricultural financing mechanisms have been put in place in cashew production. This study aimed at identifying the determinants of access to finance by cashew producers. To this end, socioeconomic data was collected through a survey of 160 cashew producers chosen at random from two municipalities in North Benin. Descriptive statistics were used to characterize the types of funding. A binary logit regression model was estimated to determine the variables influencing access to credit by cashew producers. The main results of this study indicate three types of financing obtained by cashew producers, which are financed by various actors. In addition, gender, age, membership of an organization,
\end{abstract}


contacts with extension services and training in cashew production determine access to agricultural credit in northern Benin. Finally, the promotion of this financing in the study area will make it possible to regulate the capacity to introduce agroforestry on the farm, all of which contributes to the improvement of living conditions and soil protection.

(C) 2021 International Formulae Group. All rights reserved.

Keywords: Agricultural financing, financial mechanism, N'Dali, Tchaourou.

\section{INTRODUCTION}

Le développement de filières d'exportation performantes comme la filière anacarde considérée parmi les plus prometteuses en Afrique constitue un important levier en termes d'amélioration du commerce international, de création de valeur ajoutée, d'emplois et de lutte contre la pauvreté (Ndiaye, 2019). Au Bénin, cette filière est une alternative économiquement intéressante qui représente le deuxième produit agricole d'exportation du pays après le coton (MAEP, 2017). De plus, elle génère des revenus aussi bien pour les planteurs que pour les autres acteurs de la filière (commerçants, transformateurs, exportateurs, etc.) et pour l'Etat (Assogba et al., 2017). Ainsi, la promotion de la filière anacarde devient une source de création d'emploi, de diversification des sources de revenus et des activités féminines qui ont stimulé le développement du secteur agroindustriel et la réduction de l'exode rural (Ogouvide et Sodjinou, 2012). La filière compte plus de 200000 acteurs qui assurent les fonctions de production, de transformation et de commercialisation au sein des différentes chaînes de valeurs contenues dans la filière. En effet, les différents acteurs intervenant interagissent à travers les différentes relations d'affaires qui les lient au sein des différentes chaines de valeurs ajoutées (CVA) existantes dans la filière (Gogohounga et al., 2019). Les actions de promotions menées jusque-là sont nombreuses et se situent aussi bien au niveau de la production que de la transformation, se traduisant dans ce dernier par l'installation d'un certain nombre d'unités de transformation au nord du Bénin (Akounnou et al., 2019).
Malgré les multitudes avantages que présente la filière anacarde, la productivité des anacardiers demeure très faible (Adjobo et al., 2020). Cette faible productivité a des impacts significatifs sur les conditions de vie des producteurs (Awo, 2019). Ce qui condamne les producteurs, premier acteur des CVA de la filière, à demeurer dans la pauvreté avec des faibles revenus agricoles. Cela ne permet aux producteurs de jouir de diverses opportunités de la filière comme les autres acteurs (transformateurs, commerçants, exportateurs). Par ailleurs, l'amélioration de la productivité agricole passe par l'utilisation d'équipements modernes, d'intrants améliorés, de mécanisation, de formation et d'organisation de l'ensemble des acteurs de la chaîne de valeur (Muayila et al., 2018). A cet effet, le manque d'accès au financement dans ce secteur limite les producteurs dans leurs accès au paiement des salaires de la main d'œuvre nécessaire pour les différentes opérations culturales (Flore et Omenguele, 2019). Ils ont également un accès insuffisant aux informations sur les opportunités de marché (Adjobo et al., 2020) et font face à d'autres difficultés, vu que la durée d'entrée en production des arbres après la plantation dépasse un an (INSAE, 2009). Ces changements technologiques et innovations ne peuvent se diffuser sans un financement accru mise en place et adapté aux besoins et spécificités des agriculteurs (Sourou et al., 2016; Muayila et Tollens 2012). BruléFrançoise et al. (2016), (Ribier et Gabas; (2016), Karlan et al. (2014) ont montré que l'inadéquation entre l'offre et la demande de crédit agricole est l'une des raisons de la faiblesse de la productivité agricole dans les 
pays en développement, particulièrement en Afrique. Ceci est dû à l'obligation de fournir des garanties qui exclut d'office une partie des producteurs. De plus, le nouvel objectif de développer les «chaînes de valeur » incite les structures publiques de prêt à cibler les petites et moyennes entreprises agroalimentaires (Ribier et Gabas, 2016 ; Mondedji et al., 2015). Ainsi, le crédit agricole reste structurellement lié aux dispositifs de subventions publiques (crédit de campagne, etc.), eux-mêmes largement fléchés vers les filières dites stratégiques (Daoudi, 2016), notamment la filière coton. Pour les autres filières, notamment celles bien intégrées aux marchés comme les filières anacarde, soja, maïs, ananas, le financement informel joue un rôle très capital dans toutes les formes au Bénin. L'accès au financement informel reste limité dans le rang de certains producteurs du nordBénin. Face à ces défis, les producteurs d'anacarde soucieux du devenir de leur exploitation agricole et visant le bien être de leur ménage ont donc recours aux financements agricoles en particulier le crédit agricole. D'après Fafeh (2009), les financements agricoles offrent aux producteurs un marché garanti, un crédit et une assistance technique, pouvant leur permettre de s'ouvrir aux marchés. Le crédit, fourni par des institutions financières publiques ou privées et par les usuriers, est un élément clé d'un écosystème complet, mêlant acteurs publics et privés dans de multiples domaines (recherche agronomique, enseignement agricole, conseil technique et économique, organisation des agriculteurs sous forme de coopératives et d'interprofessions, gestion des risques agricoles, services sociaux) en milieu rural (Brulé-Françoise et al. (2016).

Toutefois, notons que certains producteurs n'ont pas accès aux financements agricoles en particulier le crédit agricole. Dans le processus de l'accès aux financements agricoles, plusieurs paramètres définissent l'accès à l'un des mécanismes de financements agricoles par les producteurs d'anacarde. La présente étude détermine les paramètres influençant l'accès aux financements agricoles en particulier le crédit agricole par les producteurs d'anacarde des communes de Tchaourou et de N'Dali.

\section{MATÉRIEL ET MÉTHODES Zone d'étude}

L'étude a été conduite dans le Nord du Bénin précisément dans le département du Borgou. Le choix de ces zones part du fait que les conditions agro écologique de ces départements sont très favorables à la culture de l'anacardier (MAEP, 2017). L'aire favorable de production de l'anacarde au Bénin couvre principalement les régions du centre et du nord Bénin, (Zoumarou et al., 2016). Le Nord-Bénin est caractérisé par deux saisons ; une saison sèche et une saison pluvieuse, le Centre par deux saisons pluvieuses (une grande et une petite) et deux saisons sèches (une grande et une petite). En effet, ce département fait partie des six (06) départements où l'anacarde (Anacardium Occidentale) est une espèce spontanée, utilisée pour le reboisement et de plus en plus cultivé pour son fruit : la noix de cajou. Ainsi, les communes de Tchaourou et de N'Dali ont été retenues pour mener cette étude. Les villages retenus sont ceux où l'anacarde occupe une place importante dans les systèmes de production. C'est ainsi que quatre (04) villages représentatifs ont été retenus (Figure 1).

\section{Echantillonnage et collecte des données}

Deux villages ont été choisis par commune pour réaliser les enquêtes. Les villages concernés ont été Tchatchou et Sebou dans la commune de Tchaourou, Tamarou et Gounin dans la commune de N'Dali. Ces villages ont été choisis, pour enquêter les producteurs d'anacarde, sur la base de leur représentativité en matière des superficies emblavées pour les plantations d'anacarde et de leur accès ou non au financement agricole. De plus, les plantations d'anacarde représentent pour ces villages une activité socioéconomique. 
Les unités d'observation sont donc tous les producteurs d'anacarde (toute catégorie confondue) dans la zone d'étude. Par village sélectionné, un échantillon de 40 enquêtés potentiels des producteurs de la filière anacarde a été constitué de manière aléatoire à partir du répertoire des producteurs obtenus auprès de la Fédération nationale des Producteurs d'Anacarde du Bénin (FENAPAB) en utilisant la table des nombres aléatoires. Ainsi, au total 160 producteurs de cajou ont été enquêtés.

Les données collectées sont relatives aux types de financements obtenus par les producteurs d'anacarde; les sources et formes de financements, les modes d'accès à l'information au financement agricole, l'expérience des producteurs en financement et leur appréciation du mécanisme de financement dans la filière anacarde; les caractéristiques socioéconomiques (sexe, âge, l'appartenance à une organisation, accès au crédit, etc.) des producteurs de la filière anacarde dans la zone d'étude.

\section{Modélisation des déterminants de l'accès au crédit}

L'approche utilisée dans l'analyse des facteurs déterminants l'accès aux crédits agricoles pour le financement des activités agricoles est estimée par un modèle permettant de prédire la décision d'un producteur d'avoir accès aux crédits agricoles ou non pour financer ses activités agricoles.

Par ailleurs, les modèles utilisés dans les études d'adoption sont les modèles avec probabilité linéaire, le Logit, le Probit et le Tobit. Ces dits modèles utilisent tous des variables à choix binaire comme variable dépendante. Les modèles Probit et Logit sont utilisés lorsque la variable dépendante est dichotomique dans la plupart des études de déterminants (Yabi et al., 2016 ; Dossa et Miassi, 2018).

Suivant la nature de la distribution de $\mathrm{f}$ qui peut être normale ou logistique, l'estimation de l'équation est faite par un modèle Probit binomial ou un Logit binomial. Le modèle Logit a été retenu dans cette étude pour les trois raisons suivantes (Sossou et Fok, 2017) :

La variable expliquée (accès ou non au crédit) utilisée dans notre étude est qualitative et dichotomique.

Lorsque les variables explicatives ne sont pas normalement distribuées, les estimateurs du modèle Logit sont plus robustes que ceux obtenus par l'analyse discriminante.

Les modèles Logit permettent des calculs simples.

Le modèle Logit permet d'analyser de manière quantitative le processus de décision d'utilisation ou non une des technologies agricoles (Tene et al., 2013). Et de plus, le modèle Logit grâce à une transformation maintient la probabilité estimée entre 0 et 1 . L'équation du modèle présente comme suit:

$\mathrm{E}(\mathrm{Yi})=\mathrm{P}(\mathrm{Yi})=\frac{e^{\alpha+\beta X i}}{1+e^{\alpha+\beta X i}}$

Lorsque le producteur n'a pas accès aux crédits agricoles, la probabilité devient à cet effet :

$\mathrm{P}($ non- adoption $)=1-\mathrm{P}(\mathrm{Yi})=\frac{1}{1+e^{\alpha+\beta X i}}$

Où :

$\mathrm{P}(\mathrm{Yi})$ : La probabilité pour un individu i d'adopter le crédit agricole ; $\mathrm{P}(\mathrm{Yi})=1$ si la technologie est adoptée et 0 si la technologie n'est pas adoptée.

$e:$ La fonction exponentielle; Yi : la variable expliquée; l'adoption du crédit agricole ;

$\beta$ : Le vecteur des paramètres à estimer dont le signe permet l'interprétation des résultats ;

$\alpha$ : La constante

$\mathrm{Xi}$ : caractéristique de l'individu i ; il représente le vecteur des variables explicatives Avec $X=\beta_{0^{+}} \beta_{1}$ AGE $+\beta_{2} \mathrm{SEXE}+\beta_{3}$ DISIMF $+\beta_{4}$ PACTIS $+\beta_{5} \quad$ PREIMF $+\beta_{6}$ CVULG $+\beta_{7}$ APARORG $+\beta_{8}$ FORMCAJU + $\beta_{9}$ VENCNTRA $+\beta_{10}$ NINSTR $+\epsilon$

(Tableau 1) 


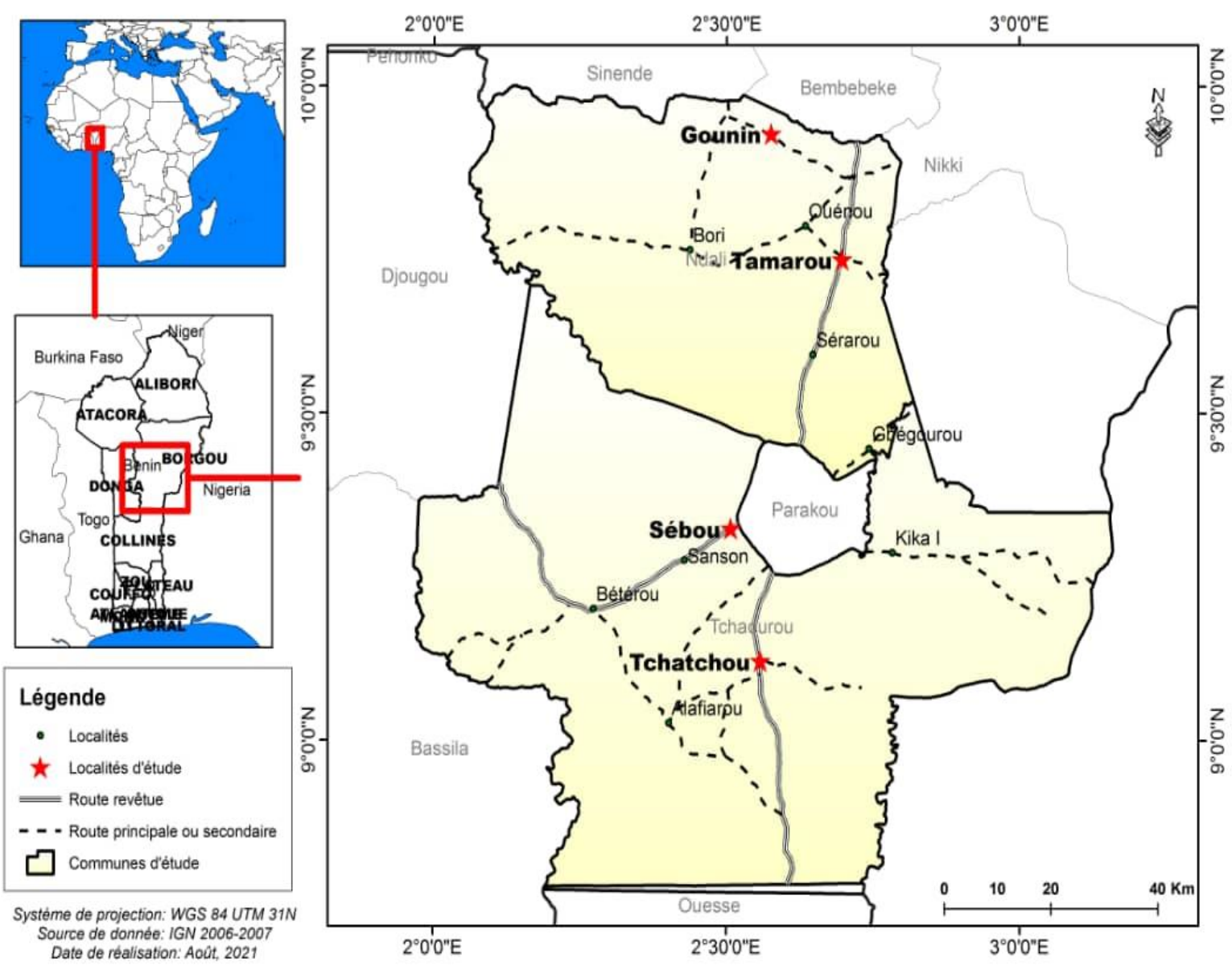

Figure 1: Carte de la zone d'étude.

Tableau 2: Variables explicatives introduites dans les modèles de régression Logit.

\begin{tabular}{lcc}
\hline Variables introduites dans le modèle & Modalités & Signe attendu \\
\hline Distance entre les IMFs et le ménage (DISIMF) & - & + \\
Présence des IMFs (PREIMF) & Non $=0 ;$ Oui $=1$ & + \\
Age (AGE) & - & $+/-$ \\
Sexe (SEXE) & Non $=0 ;$ Oui $=1$ & + - \\
Appartenance à une organisation (APARORG) & Non $=0 ;$ Oui $=1$ & + \\
Contact avec un service de vulgarisation (CVULGA) & Non $=0 ;$ Oui $=1$ & + \\
Formation sur la production d'anacarde & Non $=0 ;$ Oui $=1$ & - \\
(FORMCAJU) & & + \\
Vente sous contrat (VENCNTRA) & Non $=0 ;$ Oui $=1$ & - \\
Nombre d'année de scolarisation (NINSTR) & - & $+/-$ \\
Possession d'activité secondaire (PACTIS) & Non $=0 ;$ Oui $=1$ & \\
\hline
\end{tabular}




\section{RÉSULTATS}

Les producteurs d'anacarde du Borgou au Nord-Bénin ont accès aux crédits par plusieurs mécanismes de financements. Ces crédits leur permettent de faire face à beaucoup de contraintes pour épargner en majorité les plantations des risques pouvant entraver la productivité des anacardes.

\section{Mécanisme et accès au financement}

Trois types de financements sont obtenus par les producteurs d'anacarde dans la zone d'étude. Il s'agit de Crédit de production/entretien de plantation, de Crédit de précampagne/crédit pré-collecte et l'épargne/fonds propres.

\section{Crédit de production/entretien de plantation}

Le crédit de production ou l'entretien des plantations est un financement destiné exclusivement pour la production et/ou à l'entretien des plantations afin de générer des revenus des activités, que ce soit des activités de court terme comme le petit commerce ou des investissements à long terme comme l'amélioration des outils agricoles ou la terre. Ce type de financement épargne la plupart des temps les plantations des feux de brousses et permet aux producteurs de faire l'entretien des plantations à une bonne date afin d'obtenir de meilleure productivité. Dans la zone d'étude, les résultats obtenus révèlent que $60,9 \%$ des producteurs d'anacarde enquêtés bénéficient de ce crédit. Dans la commune de N'Dali, les producteurs $(42,2 \%)$ bénéficient ce crédit plus que les producteurs de la commune de Tchaourou. Il faut notifier que ce type de crédit est accordé aux producteurs avant le démarrage de la campagne anacarde précisément entre le mois d'août et octobre ont-ils déclaré les enquêtés.

\section{Crédit de précampagne/crédit pré-collecte}

Le crédit de précampagne/crédit précollecte est un crédit qui permet de gérer les risques liés à la production agricole notamment dans les régions marginalisées et donc l'incertitude des revenus agricoles contribuent à la vulnérabilité des moyens de survie qui constituent des menaces pour la consommation. Le crédit serait nécessaire pour réguler un tant soit peu les déficits de consommation. Cela permettra aux producteurs de mieux organiser leur campagne et bien planifier la collecte des noix. Seuls $(8,1 \%)$ des producteurs de la commune de N'Dali ont accès à ce type de crédit dans la zone d'étude. Par conséquent, ce type de crédit ne s'octroie pas dans la commune de Tchaourou.

\section{Epargne/fonds propres}

L'épargne n'est rien d'autres que le fond propre du producteur déposé auprès d'une institution de microfinance ou une structure ou un particulier. Il joue plusieurs rôles importants bien qu'étant encore négligé par certains programmes de réduction de la pauvreté. En renonçant à la consommation, les ménages augmentent leurs options dans le futur, aussi bien pour l'investissement que la consommation. L'épargne protège contre les déficits futurs, réduit la vulnérabilité et permet un investissement futur pour les activités de production. Des résultats de nos analyses, 42,9\% des producteurs de la zone d'étude ont recours à ce crédit pour financer leurs activités au sein des exploitations d'anacarde. Ce crédit est plus adopté dans la commune de Tchaourou $(35,4 \%)$ que dans la commune de N'Dali $(7,5 \%)$.

\section{Sources et formes de financement Sources de financement}

Les producteurs d'anacarde ont obtenu des financements auprès des personnes physiques et morales pour leurs diverses activités pour la campagne anacarde de 2017 2018. Le Tableau 2 montre que 19,4\% des producteurs sont financés par les IMFs présentes dans leurs communes respectives, (soit 3,9\% à Tchaourou et $15,5 \%$ à N'Dali). Une minorité $(1,3 \%)$ des producteurs sont financés par les structures de vulgarisation (URPA, BeninCajù, FENAPAB, etc.) et seulement dans la commune de Tchaourou. Les tontiniers et les usuriers financent $12,9 \%$ et $11 \%$ des producteurs d'anacarde respectifs à Tchaourou et à N'Dali. 4,5\% des producteurs bénéficient leur financement auprès des proches et amis. Plus que la moitié (51\%) des producteurs ont obtenu leur financement par 
autres sources (épargne, fonds propres, revenu extra-agricole, etc.).

Les IMFs tels que la CLCAM $(0,6 \%)$, PADME $(16,1 \%)$, CAVECA $(0,6 \%)$, et Agrifinance $(0,6 \%)$ sont ceux auprès desquels les producteurs ont obtenu leur financement. Quant aux structures de vulgarisation d'appui, seuls Bénincajù et URPA assistent les producteurs de la zone d'étude.

\section{Formes de financement}

Les financements sont octroyés aux producteurs sous différentes formes dans la zone d'étude. Trois formes de financements sont observées à savoir: le financement en nature, financement en espèce et subventions.

Il ressort du Tableau 2 que peu des producteurs $(26,1 \%)$ bénéficient le financement en nature dans la zone d'étude. Les producteurs de N'Dali $(23,6 \%)$ bénéficient cette forme de financement plus que les producteurs de la commune de Tchaourou $(2,5 \%)$. En ce qui concerne le financement en espèce, $75,8 \%$ des producteurs en bénéficient dans la zone d'étude. $47,2 \%$ à Tchaourou et $28,6 \%$ à N'Dali. Quant à la subvention, il est uniquement bénéficié par $3,1 \%$ des producteurs d'anacarde de la commune de Tchaourou.

Le montant moyen obtenu par les producteurs d'anacarde qui ont opté pour le financement en espèce est de 46 164,60 FCFA avec une durée de crédit de 2 mois en moyenne pour la campagne d'anacarde de 2017-2018. Ce montant est presque égal dans les deux communes. Il est de même pour la durée du crédit (Tableau 2).

\section{Mode d'accès à l'information au financement agricole}

Les producteurs ont adhéré au financement agricole par plusieurs modes dans le département du Borgou au Nord-Bénin. Il ressort de nos analyses que $5 \%$ des producteurs ont accès à l'information au financement par sensibilisation et uniquement à N'Dali. Très peu $(11,8 \%)$ des producteurs ont accès à l'information au financement par le biais d'un ami (soit $6,2 \%$ et $5,6 \%$ des producteurs de Tchaourou et de N'Dali respectifs). Certains producteurs $(23 \%)$ ont adhéré au financement par leur coopérative (soit 13,7\% à Tchaourou et $9,3 \%$ à N'Dali) à laquelle ils appartiennent et d'autres producteurs (13\%) ont adhéré par le biais d'un parent $(7,5 \%$ à Tchaourou et $5,6 \%$ à N'Dali). Une majorité $(47,2 \%)$ des producteurs de la zone d'étude ont adhéré au financement par autres modes (motivation personnelle, recherche de financement, etc.) à raison de $23 \%$ à Tchaourou et $24,2 \%$ à N'Dali. Le test d'indépendance de chi 2 de Pearson $\left(\chi^{2}=9,85\right.$; $\mathrm{ddl}=4 ; \mathrm{p}=0,04)$ montre que ces modes d'adhésion sont liées aux différentes manières dont chaque mode est utilisé dans la zone d'étude afin que les producteurs puissent opter aux différents mécanismes de financement qui lui sont proposés.

Par ailleurs, il y a des raisons qui ont amené les producteurs à adhérer aux différents modes de financement dans leur commune respective. Il ressort de nos analyses que la raison majeure d'adhésion au financement agricole est l'entretien des plantations d'anacardes (62,7\% des enquêtés). L'accès aux crédits est l'une des raisons d'adhésion pour $12,4 \%$ des producteurs. Très peu $(1,2 \%)$ des producteurs ont adhéré au financement agricole à cause de l'assurance de la production et $34,8 \%$ des producteurs ont adhéré pour autres raisons à savoir : complément de fonds, épargne, Facilité les préparatifs de la campagne d'anacarde, etc.

\section{Expérience en financement et appréciation du mécanisme de financement}

Les producteurs de la zone d'étude ont une expérience moyenne de 3,20 $( \pm 4,23)$ ans avec un minimum de 1 an et un maximum de 35 ans dans l'adoption du financement agricole quels que soient les mécanismes. Les producteurs de la commune de Tchaourou (2,54 $\pm 4,74$ ans) sont moins expérimentés que les producteurs de N'Dali (3,86 $\pm 3,56$ ans) en financement agricole. Par ailleurs, les producteurs ont apprécié les mécanismes de financement auxquels ils adhèrent respectivement. Alors, $25,5 \%$ des producteurs sont très faiblement satisfait du mécanisme de financement qui leur est appliqué. Très peu des producteurs de N'Dali $(0,6 \%)$ et peu des producteurs de Tchaourou $(24,8 \%)$ sont très 
faiblement satisfait. La majorité $(42,2 \%)$ des producteurs est moyennement satisfaite des mécanismes du financement qui leur sont proposés soit $9,3 \%$ à Tchaourou et $32,9 \%$ à N'Dali. Par contre, $8,7 \%$ des producteurs sont très fortement satisfait $(3,1 \%$ à Tchaourou et $5,6 \%$ à N'Dali) et $14,9 \%$ sont fortement satisfaits avec $10,6 \%$ à Tchaourou et $4,3 \%$ N'Dali respectifs chacun des mécanismes du financement. Les producteurs de Tchaourou sont fortement satisfaits $(10,6 \%)$ tandis que les producteurs de N'Dali sont moyennement satisfaits $(32,9 \%)$.

\section{Déterminants de l'accès aux crédits agricoles par les producteurs d'anacarde}

Le crédit agricole constitue un levier très important dans le processus de la promotion $\mathrm{du}$ secteur agricole et de l'amélioration des conditions de vie des producteurs. La majorité des populations réside en milieu rural et pour assurer la sécurité alimentaire et réduire la pauvreté, il est important de promouvoir l'essor de l'agriculture dans ces zones et de fournir aux ruraux un meilleur accès à la terre, à l'eau, au crédit, à la santé et à l'éducation (Aïhounton et al., 2016). Le rôle des structures d'appuis techniques et financières (IMFs, ONGs, projets, etc.) dans le développement économique et social du monde rural par le levier que représente l'intermédiation financière et technique, rend naturel l'intérêt croissant à étendre l'accès à de tels services dans les zones rurales défavorisées. Ainsi, les producteurs d'anacardes des zones rurales pour la plupart, ont recours aux institutions financières afin d'avoir accès au crédit financier agricole dans le but d'accroître ou d'améliorer leur production. Cette partie s'intéresse donc aux différents facteurs qui déterminent l'accès aux crédits agricoles par les producteurs d'anacarde.

Les variables introduites dans le modèle de régression Logit permettent d'expliquer $37,6 \%$ des variations des variables respectives de l'accès aux crédits agricoles par les producteurs d'anacarde dans les communes de N'Dali et de Tchaourou au Nord-Benin. En outre, le test d'indépendance de chi-deux traduit que la matrice de corrélation est significative (Chi2 : 49,85; dll : 10 ; p: 0,000). Ce qui indique que la modélisation utilisée dans le cadre de l'étude est bien justifiée. L'accès aux crédits agricoles par les producteurs d'anacarde dans les communes de N'Dali et de Tchaourou est déterminé par des facteurs tels que le sexe, l'âge, l'appartenance à une organisation, le contact avec un service de vulgarisation et la formation sur la production d'anacarde.

L'âge détermine positivement l'accès aux crédits agricoles au seuil de $10 \%$. Plus les producteurs sont âgés, plus ils ont recours aux crédits agricoles pour les diverses opérations des plantations d'anacarde.

Le sexe influence positivement l'accès aux crédits agricoles au seuil de 5\%. Alors, les hommes de la zone d'étude sont plus intéressés aux crédits agricoles que les femmes du fait que l'activité agricole révèle de la compétence des hommes et la quasi-totalité de ces derniers s'adonnent à cette activité en milieu rural. De plus, les femmes sont très réticentes à la prise des risques dans l'exercice des activités agricoles vis-à-vis des microfinances où elles pratiquent diverses opérations.

L'appartenance $\grave{a}$ une organisation détermine positivement à $1 \%$ l'accès aux crédits agricoles par les producteurs d'anacarde $\mathrm{du}$ nord-Bénin. Les producteurs qui appartiennent à une organisation ont plus de la facilité à obtenir de crédit agricole vue que les crédits s'accordent aux producteurs en groupement sous réserve des cautions solidaires pour garantir le remboursement effectif des fonds empruntés et limiter les risques d'insolvabilité.

Le contact avec un service de vulgarisation impacte négativement l'accès aux crédits agricoles par les producteurs d'anacarde du nord-Bénin à 5\%. Plus les producteurs sont en contact avec les services de vulgarisation, moins ils ont accès aux crédits agricoles.

La formation sur la production d'anacarde a un effet positif sur l'accès aux crédits agricoles par les producteurs d'anacarde au seuil de $10 \%$. Le producteur ayant bénéficié d'une formation en techniques de production 
d'anacarde aura plus besoin de financement pour s'acquitter des facteurs de productions nécessaires pour la mise en application des techniques reçues au cours de la formation.

Quant à la distance entre les IMFs et le ménage, la présence des IMFs, la Vente sous contrat, l'expérience en agriculture et la possession d'activité secondaire, ces variables n'ont aucun impact significatif sur la détermination de l'accès aux crédits agricoles dans la production d'anacarde au Nord-Bénin. Cela s'explique par le fait que l'accès aux crédits agricoles se trouve moins contraignant pour les producteurs qui ont vendu leur noix sous contrat qu'à ceux qui ne l'ont pas fait. De même, il ne s'agit pas d'avoir de l'expérience en agriculture ou de posséder une activité secondaire ou avoir la présence des IMFs dans sa localité pour avoir accès aux crédits agricoles (Tableau 3).

Tableau 2: Formes et sources de financement.

\begin{tabular}{|c|c|c|c|c|c|c|}
\hline \multicolumn{7}{|c|}{ Sources de financement } \\
\hline Zone d'étude & IMF & $\begin{array}{c}\text { Structure de } \\
\text { vulgarisation }\end{array}$ & Tontine & Usuriers & Proches & Autres \\
\hline Tchaourou & 3,9 & 1,3 & 12,9 & 2,6 & 2,6 & 25,8 \\
\hline N'Dali & 15,5 & 0 & 0 & 8,4 & 1,9 & 25,2 \\
\hline Ensemble & 19,4 & 1,3 & 12,9 & 11 & 4,5 & 51 \\
\hline \multicolumn{7}{|c|}{ Formes de financement (\%) } \\
\hline & \multicolumn{2}{|c|}{ Financement en nature } & \multicolumn{2}{|c|}{ Financement en espèce } & \multicolumn{2}{|c|}{ Subventions } \\
\hline Tchaourou & \multicolumn{2}{|r|}{2,5} & \multicolumn{2}{|c|}{47,2} & \multicolumn{2}{|c|}{3,1} \\
\hline N'Dali & \multicolumn{2}{|r|}{23,6} & \multicolumn{2}{|c|}{28,6} & \multicolumn{2}{|c|}{0} \\
\hline Ensemble & \multicolumn{2}{|r|}{26,1} & \multicolumn{2}{|c|}{75,8} & \multicolumn{2}{|c|}{3,1} \\
\hline
\end{tabular}

Période d'accord du crédit aux producteurs (\%)

Avant la campagne Au cours de la En fin de contrat
campagne

\begin{tabular}{lllc}
\hline Tchaourou & 41,6 & 6,8 & 1,9 \\
\hline N'Dali & 48,4 & 1,2 & 0,0 \\
\hline Eensemble & 90,1 & 8,1 & 1,9 \\
\hline Test de Khi2 & & $\chi^{2}=10,06 ;$ ddl $=2 ; \mathrm{P}=0,007$ \\
\hline
\end{tabular}

Durée et période d'accord du crédit aux producteurs

\begin{tabular}{lcc}
\hline & $\begin{array}{c}\text { Si financement en } \\
\text { espèce, Montant obtenu } \\
\text { (FCFA) }\end{array}$ & $\begin{array}{c}\text { Durée du crédit (en } \\
\text { mois) }\end{array}$ \\
\hline Tchaourou & $46821(65964,64)^{*}$ & $1,95(2,87)^{*}$ \\
\hline N'Dali & $45500(49883,41)$ & $2,34(2,63)$ \\
\hline Total & $46164,60(58350,07)$ & $2,14(2,75)$ \\
\hline Test de T-Student & $\mathrm{t}=-0,891 ; \mathrm{ddl}=159 ;$ & $\mathrm{t}=0,143 ; \mathrm{ddl}=159 ;$ \\
& $\mathrm{P}=0,374$ & $\mathrm{P}=0,886$
\end{tabular}

*les valeurs entre ( ) représentent l'écart-types 
Tableau 3: Récapitulatif du modèle de régression logit.

\begin{tabular}{|c|c|c|c|}
\hline Variables introduites dans le modèle & Coefficient & Erreur Standard & $\mathbf{P}>|\mathbf{z}|$ \\
\hline $\begin{array}{l}\text { Distance entre les IMFs et le ménage } \\
\text { (DISIMF) }\end{array}$ & 0,02 & 0,01 & 0,13 \\
\hline Présence des IMFs (PREIMF) & 0,67 & 0,59 & 0,25 \\
\hline Age (AGE) & $0,04 *$ & 0,02 & 0,07 \\
\hline Sexe (SEXE) & $2,13 * *$ & 0,96 & 0,02 \\
\hline $\begin{array}{l}\text { Appartenance à une organisation } \\
\text { (APARORG) }\end{array}$ & $2,83^{* * *}$ & 0,69 & 0,00 \\
\hline $\begin{array}{l}\text { Contact avec un service de } \\
\text { vulgarisation (CVULGA) }\end{array}$ & $-2,53 * *$ & 1,16 & 0,02 \\
\hline $\begin{array}{l}\text { Formation sur la production } \\
\text { d'anacarde (FORMCAJU) }\end{array}$ & $1,69 *$ & 1,01 & 0,09 \\
\hline $\begin{array}{l}\text { Vente sous contrat } \\
\text { (VENCNTRA) }\end{array}$ & 1,30 & 1,02 & 0,20 \\
\hline Expérience en agriculture (ExpA) & $-0,03$ & 0,02 & 0,18 \\
\hline $\begin{array}{l}\text { Possession d'activité secondaire } \\
\text { (PACTIS) }\end{array}$ & $-0,54$ & 0,45 & 0,22 \\
\hline Constante & $-5,40 * * *$ & 1,50 & 0,00 \\
\hline
\end{tabular}

\begin{tabular}{cc}
\hline \multicolumn{2}{c}{ Résumé du modèle logit } \\
\hline Nombre d'observation \\
Log vraisemblance & 160 \\
Chi-deux & 155,13 \\
Probabilité >chi2 & 49,85 \\
Pseudo R2 & 0,00 \\
Dll & 0,37 \\
\hline
\end{tabular}

*** : valeur significative à $1 \%(\mathrm{P} \leq 0,01) ; *^{* *}$ : valeur significative à $5 \%(0,01<\mathrm{P} \leq 0,05) ; *$ : valeur significative à $10 \%(0,05<$ $\mathrm{P} \leq 0,10)$.

\section{DISCUSSION}

Plusieurs mécanismes de financements ont été adoptés par les producteurs d'anacarde dans la zone d'étude. L'épargne et/ou fonds propres est le mécanisme de financement le plus adopté par les producteurs de la zone d'étude. Très peu de ces producteurs adoptent ou ont accès aux crédits de la production/crédit d'entretien des plantations, crédits de précampagne et/ou crédit de pré-collecte. Cela s'explique par le fait que les producteurs disposent des ressources suffisantes pour investir dans la production d'anacarde. Ces ressources sont parfois obtenues des revenus des autres cultures ou des activités extraagricoles. La plupart des producteurs veulent être indépendants des IMFs à cause de leurs dérangements et pressions sur le délai de remboursement relativement très court. Du fait que le crédit sollicité n'est souvent pas accordé au moment où ils sont dans le besoin pour la réalisation des activités programmées. D'après (Dossa et Miassi, 2018), les petits producteurs sous contrat sont exposés à des risques d'endettement et de perte d'autonomie. Les risques que présente le contrat agricole sont liés à la crainte du producteur de ne pas pouvoir rembourser le crédit obtenu. Les diverses structures d'accompagnement des producteurs dans le financement agricole (toutes formes confondues) ne tiennent pas compte des périodes de besoins de ces derniers. Sur ce, (Sossou et Fok, 2017) avaient perçu que les besoins en services financiers des agriculteurs ruraux portent sur diverses formes de crédit (de court terme pour la campagne, l'embauche, la soudure ; de moyen terme pour l'équipement; de long terme pour l'équipement lourd, les 
plantations, etc.) et évoluent suivant chaque catégorie de classe d'exploitants.

En effet, l'accès au crédit ne peut aider le pauvre à sortir de sa pauvreté que lorsque ce dernier est économiquement actif, possède une sécurité économique. Ceci se ferait grâce à l'amélioration de la productivité ou la création de nouvelles sources de moyen d'existence (Assogba et al., 2017). Plusieurs études ont montré que les facteurs déterminant l'adoption d'une technologie ou une innovation en milieu paysan sont liés non seulement à la technologie mais aussi aux conditions socioéconomiques et culturelles du paysan. Toutefois, le sens de cette influence varie d'une thématique à une autre et selon le contexte. Ainsi, des résultats de la présente étude, l'âge a un effet positif sur l'accès aux crédits agricoles. Les producteurs plus âgés disposent peu de ressources pour financer les activités liées aux plantations car ils disposent peu de forces physiques et aussi de la main d'œuvre suffisante. Du fait que les enfants ont pour la plupart l'âge de fonder leur propre ménage. Ce résultat confirme celui de (Sossou et Fok, 2017) pour qui les institutions de financement préfèrent prêter aux exploitants les plus âgés voire les plus expérimentés. Pour eux, l'âge est un indicateur de maturité qui permet de savoir à quel seuil l'emprunteur est capable de rembourser le crédit avec un taux d'intérêt à la fin de l'échéance. Ceci est en concordance avec les résultats de Sery (2012) qui avait trouvé qu'en Côte d'Ivoire, les IMF n'accordent de crédit qu'aux femmes dont leurs âges sont compris entre 21 ans et 45 ans. Ainsi, les jeunes ont tendance à épargner tandis que les personnes âgées sont à la recherche du financement pour investir dans leurs diverses activités (Flore et Omenguele, 2019).

Le sexe détermine positivement et significativement l'accès aux crédits agricoles. Alors, les hommes de la zone d'étude sont plus intéressés aux crédits agricoles que les femmes du fait que l'activité agricole révèle de la compétence des hommes et la quasi-totalité de ces derniers s'adonnent à cette activité en milieu rural. De plus, les femmes sont très réticentes à la prise des risques dans l'exercice des activités agricoles vis-à-vis des microfinances où elles pratiquent diverses opérations. Le résultat est contraire à celui de (Mayoukou et Kertous, 2015) qui avaient obtenu que le sexe ne détermine pas l'accès aux crédits agricoles au Bénin donc par conséquent, il n'est pas un facteur de discrimination entre les hommes et les femmes pour l'accès au crédit au niveau des Institutions de microfinance. Par ailleurs, ce résultat confirme les études qui avaient révélé que les femmes sont lésées dans l'accès aux services financiers (Leite et al., 2019). Nsengiyumva et Mayoukou (2018) avaient obtenu que la plupart des bénéficiaires des microcrédits sont des hommes (69,33\%). Par ailleurs, l'appartenance à une organisation a un impact positif et significatif sur l'accès aux crédits agricoles par les producteurs d'anacarde du nord-Bénin. Les producteurs qui appartiennent à une organisation ont plus de la facilité à obtenir de crédit agricole vue que les crédits s'accordent aux producteurs en groupement sous réserve des cautions solidaires pour garantir le remboursement effectif des fonds empruntés et limiter les risques d'insolvabilité. Ces résultats corroborent celui de Sossou et al. (2017) qui ont obtenu que l'appartenance à une association est un déterminant pertinent car la probabilité d'accorder le crédit aux demandeurs membres d'un groupement de solidarité financière est plus élevée que pour les non membres. Selon ces auteurs, au Bénin, le crédit agricole n'est pas individuel du point de vue de la banque, qui ne reconnait en pratique que le groupement. Ces résultats concordent avec ceux de Gogohounga et al. (2019), et ont obtenu que les producteurs qui appartiennent à un groupement mieux organisé, ont tendance à croire que les modèles d'affaires dans la filière anacarde leur permettent d'avoir accès facilement au crédit. Il est de même pour Muayila et al. (2018) qui ont trouvé que les producteurs membres des organisations paysannes ont une forte propension à accéder au crédit-intrant que les non membres. Ces résultats apparaissent contradictoires avec ceux de Nsengiyumva et Mayoukou (2018) qui ont obtenu que le fait d'être dans des groupements agricoles solidaires influe négativement à l'accès au crédit agricole dans leur étude sur les déterminants du financement des micro- 
entreprises agricoles par les institutions de microfinance au Burundi. Pour ceux, leurs résultats traduiraient les risques inhérents aux activités agricoles. Ces risques sont plus prononcés lorsque la solidarité entre les membres du groupe solidaire n'est pas battue sur les bases solides. Or, les exploitants solidaires, avec l'existence d'un fonds de garantie mis en place (caution solidaire) sont souvent battus sur la solidarité. Aussi, la formation sur la production d'anacarde impacte positivement l'accès aux crédits agricoles par les producteurs d'anacarde. Elle permet aux paysans d'avoir une connaissance de la technologie et de son utilisation. De ce fait, une des clefs de l'autonomie des producteurs, c'est d'avoir la maîtrise des compétences qui leur sont directement nécessaires (Koffi et Oura, 2019). La formation des agriculteurs est aussi une des composantes du financement agricole car dans des grands enjeux du développement agricole, chaque acteur joue sa partition en fonction de ses intérêts propres et aucun mécanisme ne permet d'assurer que certains besoins prioritaires seront bien couverts (Ribier et Gabas, 2016).

\section{Conclusion}

Cette étude a permis l'identification des déterminants de l'accès au financement agricole. Ainsi, cinq variables tels que le sexe, l'âge, l'appartenance à une organisation, le contact avec un service de vulgarisation et la formation sur la production d'anacarde déterminent l'accès aux crédits agricoles au Nord-Bénin. Par ailleurs, trois types de financements sont obtenus par les producteurs d'anacarde dans la zone d'étude. Il s'agit de Crédit de production/entretien de plantation, de Crédit de précampagne/crédit pré-collecte, et l'Epargne/fonds propres. Les financements sont octroyés aux producteurs sous différentes formes à savoir: le financement en nature, financement en espèce et subventions. Les producteurs ont apprécié les mécanismes de financement auxquels ils adhèrent respectivement. Bien qu'aucun des producteurs enquêtés n'opte pour l'assurance agricole dans la zone d'étude, elle est une option très porteuse en ce sens qu'elle contribue non seulement à augmenter la capacité des producteurs à plus planter des arbres pour la sécurité de leur terre et surtout à lutter contre la pauvreté dans le contexte d'obtenir des revenus plus stables à la longue. Il est de même pour les autres mécanismes de financement identifié. La promotion de chacun de ces financements dans la zone d'étude permettra de réguler la capacité à introduire l'agroforesterie dans leur exploitation agricole, tout ceci contribue à l'amélioration des conditions de vie et la protection des sols.

\section{CONFLIT D'INTERETS}

Les auteurs de ce manuscrit déclarent qu'ils n'ont aucun conflit d'intérêts.

\section{CONTRIBUTIONS DES AUTEURS}

AJM a financé la collecte des données, AJM et ON ont analysé les données et écrit le manuscrit, YAJ a supervisé tout le travail, tous les co-auteurs ont lu et approuvé le manuscrit final.

\section{REMERCIEMENTS}

Les auteurs remercient les chefs ménages producteurs d'anacarde des communes de N'Dali et de Tchaourou, tous les membres du LARDES pour la disponibilité lors de la collecte et l'analyse des données et d'échanges fructueux.

\section{RÉFÉRENCES}

Adjobo OMFR, Yabi JA, Gouwakinnou JY. 2020. Typologie des exploitations agricoles productrices d'anacarde au Nord et au Centre du Bénin, Glazoué, Tchaourou et Djougou. Afrique SCIENCE, 16(5): 303-316. DOI : http://www.afriquescience.net/PDF/16/5/ 25.pdf

Aïhounton DGB, Yabi JA, Bachabi FX, Yegbemey RN, Kindemin AO, Labiyi IA. 2016. Socio-economic determinants of the economic profitability of cashew nuts marketing in North-Eastern-Benin: Case study of Tchaourou municipality. International Journal of Innovation and Scientific Research, 21(1): 212-219. 
Akounnou JDF, Degla P, Idrissou L, Gantoli G. 2019. Mécanismes de financement des fournisseurs des noix d'anacarde aux unités de transformation dans le nord Benin. Agronomie Africaine, 31(2): 159172.

Assogba PN, Kokoye SEH, Yegbemey RN, Djenontin JA, Tassou Z, Pardoe J, Yabi JA. 2017. Determinants of credit access by smallholder farmers in North-East Benin. Journal of Development and Agricultural Economics, 9(8): 210-216.

Awo SJM. 2019. Mécanismes de financement et performance économique de la production de noix de cajou au Nord Bénin. Mémoire de Master Recherche, Ecole Doctorale Sciences Agronomiques et Eau, Université de Parakou, Bénin, p. 120.

Brulé-Françoise A, Faivre-Dupaigre B, Fouquet B, Tafforeau MJN, Rozières C, Torre C. 2016. Le crédit à l'agriculture, un outil-clé du développement agricole. Techniques Financières et Développement, (3): 35-52.

Daoudi A. 2016. Le financement informel du secteur maraîcher en Algérie : le cas du crédit fournisseur. Techniques Financières et Développement, 124(3) : 107-116.

Dossa FK, Miassi YES. 2018. Facteurs socioéconomiques influençant l'adoption de Coton biologique au Nord-Est du Bénin: cas de la Commune de Kandi. International Journal of Progressive Sciences and Technologies, 62 : 577-584, DOI :

http://ijpsat.es/index.php/ijpsat/article/do wnload/293/167

Fafeh A. 2009. Contrat agricole et ses effets sur les performances de production vivrière dans la commune de Kétou: Cas du Maïs (Zea mays). Mémoire pour l'obtention du diplôme d'ingénieur agroéconomiste, FSA/UAC, Bénin, p. 97.

Flore KKC, Omenguele GR. 2019. L'effet synergique des déterminants de l'accès au financement bancaire par les PME au Cameroun. Journal of Academic Finance, 10(2):84-101. DOI: https://www.scientific-
society.com/journal/index.php/AF/article /download/325/197

Gogohounga M, Labiyi IA, Coami AG, Miassi YE, Ollabodé N, Yabi JA. 2019. Caractérisation des formes de contractualisation dans la filière anacarde dans le département des collines au Benin. Agronomie Africaine, 31(2) :173186.

DOI :

https://www.ajol.info/index.php/aga/artic le/download/190369/179586

INSAE (Institut National de la Statistique et de l'Analyse Economique). 2009. Dynamique des filières d'exportation au Bénin de 1999 à 2008: Cas de quatre produits (anacarde, ananas, tabac, sucre de canne), p. 56.

Karlan D, Osei R, Osei-Akoto I, Udry C. 2014. Agricultural decisions after relaxing credit and risk constraints. The Quarterly Journal of Economics, 129(2):597-652. DOI:

https://www.nber.org/system/files/worki ng_papers/w18463/w18463.pdf

Koffi SY, Oura KR. 2019. Les facteurs de l'adoption de l'anacarde dans le bassin cotonnier de Côte d'Ivoire. Cahiers Agricultures, DOI : https://www.cahiersagricultures.fr/article s/cagri/pdf/2019/01/cagri190022.pdf

Leite RDO, Civitarese J. 2019. Microfinance pour les femmes: ya-t-il des raisons économiques? Témoignages d'Amérique latine. Bulletin Economique, 39(1) : 571580.

MAEP 2017. Plan Stratégique de Développement du Secteur Agricole (PSDSA) 2025 et Plan National d'Investissements Agricoles et de Sécurité Alimentaire et Nutritionnelle PNIASAN 2017-2021. Rapport final. Cotonou : MAEP, p. 135.

Mayoukou C, Kertous M. 2015. L'accès au crédit individuel par les clients des institutions de microfinance du Congo: une analyse des déterminants de l'autoexclusion et de l'obtention du prêt. Mondes en Développement, (1): 121-138.

DOI : https://www.cairn.info/journal-mondesen-developpement-2015-1-page-121.htm 
Muayila KH, Mofilinga BC, Mujinga K. 2018. Impact de l'accès au crédit intrant sur les performances des exploitations agricoles de Banza-ngungu. Revue Congolaise d'Economie et de Gestion, EDUPC, 2018. DOI: https://hal.archives-ouvertes.fr/hal01831853/document

Muayila KH, Tollens E. 2012. Assessing the impact of credit constraints and economic welfare of farm-household in the hinterland of Kinshasa, African Journal of Food, Agriculture, Nutrition and Development, 62: 577-584. DOI: https://www.ajol.info/index.php/ajfand/a rticle/view/77092/67554

Ndiaye IS. 2019. Analyse de la performance d'une filière d'exportation et impact dans l'atténuation des effets des changements. This paper was presented at the Conference on Climate Change and Food Security in West Africa co-organized by Université Cheikh Anta Diop de Dakar (UCAD) and Center for Development Research (ZEF), University of Bonn, on 17-18 November 2019 in Dakar, Senegal. $13 \mathrm{p}$.

Nsengiyumva T, Mayoukou C. 2018. Déterminants du financement des microentreprises agricoles par les institutions de microfinance: cas du Burundi. In : 4ème Colloque International de l'Université de Parakou.

Ogouvide TF, Sodjinou E. 2012. Analyse de la performance du marché de noix anacarde au Bénin. Rapport de performance du marché de noix d'anacarde au Bénin. PAPA/ INRAB, p. 17.

Ribier V, Gabas JJ. 2016. Vers une accentuation des disparités dans le financement de l'agriculture en Afrique de l'Ouest? Cahiers. Agriculture., 25 : 65007.

DOI :

https://agritrop.cirad.fr/582787/1/cagri16 0030.pdf

Séry A. 2012. Le microcrédit: l'empowerment des femmes ivoiriennes. Thèse de doctorat, Université Paul-Valery Montpellier III, Montpellier, p. 382.

Sossou KB, Fok M. 2017. Crédit de trésorerie et endettement des producteurs de coton: un cas au Centre-Bénin. In : Actes du Colloque de Bamako, Novembre 2017, p. 15.

Tene M, Laure G, Havard M, Temple L. 2013. Déterminants socio-économiques et institutionnels de l'adoption d'innovations techniques concernant la production de maïs à l'ouest du Cameroun. DOI : https://agritrop.cirad.fr/572422/1/docum ent_572422.pdf.

Wallis NZ, Bagnan MA, Akossou AYJ, Kanlindogbe CB. 2016. Caractérisation morphologique d'une collection de fruits d'anacardier provenant de la commune de Parakou (Bénin). International Journal of Biological and Chemical Sciences, 10(6): 2413-2422.

DOI: http://dx.doi.org/10.4314/ijbcs.v10i6.1

Yabi JA, Bachabi FX, Labiyi IA, Ode CA, Ayena RL. 2016. Déterminants socioéconomiques de l'adoption des pratiques culturales de gestion de la fertilité des sols utilisées dans la commune de Ouaké au Nord-Ouest du Bénin. International Journal of Biological and Chemical Sciences, 10(2): 779-792 DOI: https://dx.doi.org/10.4314/ijbcs.v13i7.11

Sourou BN, Yabi JA, Ouinsavi CAIN ET Sokpon N. 2016. Importance socioéconomique de la prune rouge (Haematostaphis barteri Hook F.) au Bénin. International Journal of Biological and Chemical Sciences, 10(1): 326-343.

DOI: http://dx.doi.org/10.4314/ijbcs.v10i1.25

Mondedji AD, Nyamador WS, Amevoin K, Adéoti R, Abbévi Abbey G, Koffivi Ketoh G, Glitho IA. 2015. Analyse de quelques aspects du système de production légumière et perception des producteurs de l'utilisation d'extraits botaniques dans la gestion des insectes ravageurs des cultures marâichères au Sud du Togo. International Journal of Biological and Chemical Sciences, 9(1): 98-107.

DOI: http://dx.doi.org/10.4314/ijbcs.v9i1.10. 